\title{
UNIVERSAL LOEB-MEASURABILITY OF SETS AND OF THE STANDARD PART MAP WITH APPLICATIONS
}

\author{
D. LANDERS AND L. ROGGE
}

\begin{abstract}
It is shown in this paper that for $K$-saturated models many important external sets of nonstandard analysis-such as monadic sets or the set of ail near-standard points or all pre-near-standard points or all compact points-are universally Loeb-measurable, i.e. Loeb-measurable with respect to every internal content. We furthermore obtain universal Loeb-measurability of the standard part map for topological spaces which are not covered by previous results in this direction.

Moreover, the standard part map can be used as a measure preserving transformation for all $\tau$-smooth measures, and not only for Radon-measures as known up to now.

Applications of our results lead to simple new proofs for theorems of classical measure theory. We obtain e.g. the extension of $\tau$-smooth Baire-measures to $\tau$-smooth Borel-measures, the decomposition theorems for $\tau$-smooth Baire-measures and $\tau$-smooth Borel-measures and Kakutani's theorem for product measures.
\end{abstract}

1. Introduction. In [7 and 9] Loeb described a measure construction in nonstandard analysis which gave nonstandard measure theory a fresh impetus. His construction is the following.

If $\mathscr{A}$ is an algebra on a set $X$ and $\nu:{ }^{*} \mathscr{A} \rightarrow *[0, \infty)$ is an internal finite content, then

$$
\nu_{L}(A)={ }^{\circ}(\nu(A)), \quad A \in * \mathscr{A},
$$

defines a finite measure on the algebra ${ }^{*} \mathscr{A}$, and the system $L(\nu, * \mathscr{A})=L(\nu)$ of all $A \subset{ }^{*} X$ with

$$
\sup \left\{\nu_{L}(B): * \mathscr{A} \ni B \subset A\right\}=\inf \left\{\nu_{L}(C): A \subset C \in *^{*} \mathscr{A}\right\}
$$

is the $\sigma$-field of all Carathéodory-measurable subsets of ${ }^{*} X$ with respect to $\left.\nu_{L}\right|^{*} \mathscr{A}$. The common value of the above expressions defines the unique extension of $\left.\nu_{L}\right|^{*} \mathscr{A}$ to a measure on the complete $\sigma$-algebra $L(\nu) \supset * \mathscr{A}$. This extension is also denoted by $\nu_{L} ; \nu_{L}$ is the Loeb-measure associated with $\nu$. A set $A \subset{ }^{*} X$ is universally Loeb-measurable if $A \in L(\nu)$ for all finite internal contents $\left.\nu\right|^{*} \mathscr{A}$. The system of all universally Loeb-measurable sets is denoted by $L_{u}\left({ }^{*} \mathscr{A}\right)=L_{u}$.

Received by the editors June 20, 1986.

1980 Mathematics Subject Classification (1985 Revision). Primary 28C15.

Key words and phrases. Loeb-measures, Baire- and Borel-measures, representation and extension of measures, $K$-saturated models. 
Let $(X, \mathscr{T})$ be a topological Hausdorff-space where $\mathscr{T}$ is the system of open sets. Assume that a structure is given including $X$ and the set $\mathbf{R}$ of real numbers. In this paper we consider $K$-saturated nonstandard models for these structures, where $K$ is a cardinal number with $K>\operatorname{card}(\mathscr{T})$ and $K>\boldsymbol{\aleph}_{0}$. For any $x \in X$ we call

$$
m(x)=\bigcap\{T: x \in T, T \in \mathscr{T}\}
$$

the monad of $x$. If $y \in{ }^{*} X$ and $y \in m(x)$ for some $x \in X$, we write $\operatorname{st}(y)=x$ and say that $x$ is the standard part of $y$. As $X$ is a Hausdorff-space, the standard part is uniquely determined, if it exists. Any $y$ having a standard part is called near-standard. The set of near-standard points in ${ }^{*} X$ is denoted by ns $\left({ }^{*} X\right)$.

The standard part map st:ns $\left({ }^{*} X\right) \rightarrow X$ has been investigated by several authors (see $[1,2,3,5,8,11])$. The standard part map is of great interest for various fields of applications, since in many cases it can be used as a measure preserving transformation. Often measure preserving transformations have solved problems where purely transfer-based methods failed; for more information on the importance of the standard part map see [2].

Anderson [2] has shown that for each finite Radon-measure $\mu$ defined on the Borel-algebra $\mathscr{B}(=\sigma(\mathscr{T}))$ of $X$, the standard part map is a measure preserving transformation, i.e.

$$
\begin{array}{ll}
\text { st }^{-1}(B) \in L\left({ }^{*} \mu,{ }^{*} \mathscr{B}\right) & \text { for all } B \in \mathscr{B} ; \\
\mu(B)={ }^{*} \mu_{L}\left(\mathrm{st}^{-1}(B)\right) & \text { for all } B \in \mathscr{B} .
\end{array}
$$

If $\mu \mid \mathscr{B}$ is a finite Borel-measure, fulfilling (1), then ${ }^{*} \mu_{L}{ }^{\circ} \mathrm{st}^{-1} \mid \mathscr{B}$ is a Radon-measure according to Theorem 4(ii). Hence only Radon-measures $\mu$ can be represented by $\mu={ }^{*} \mu_{L}$ o ${ }^{\circ t^{-1}}$. Therefore the methods used in the literature up to now for representing Borel-measures cannot lead beyond Radon-measures.

To handle more general measures than Radon-measures a new approach is necessary. To this aim we introduce the following concepts: If $\nu:{ }^{*} \mathscr{A} \rightarrow{ }^{*}[0, \infty)$ is a finite internal content, put for each $A \subset{ }^{*} X$

$$
\bar{\nu}(A):=\inf \left\{\nu_{L}(B): A \subset B \in \mathscr{A}^{*}\right\}, \quad \underline{\nu}(A):=\sup \left\{\nu_{L}(B): A \supset B \in \mathscr{A}^{*}\right\} .
$$

Then - by Loeb- $\bar{\nu}, \underline{\nu}$ are the outer respectively inner measures induced by $\nu_{L .} \mid L\left(\nu,{ }^{*} \mathscr{A}\right)$, and hence $\bar{\nu}(A)=\underline{\nu}(A)$ if and only if $A \in L\left(\nu,{ }^{*}\right)$.

Theorem 4(ii) shows that in a regular Hausdorff-space $\underline{\nu} \circ \mathrm{st}^{-1} \mid \mathscr{B}$ is Radon and $\bar{\nu} \circ \mathrm{st}^{-1} \mid \mathscr{B}$ is $\tau$-smooth for each finite internal content $\left.\nu\right|^{*} \mathscr{B}$. Hence only the concept of outer measures $\bar{\nu}$ can be a suitable approach for representing non-Radon-measures. Indeed it is: According to Theorem 9(ii) we have that

$$
\mu\left|\mathscr{B}={ }^{*} \bar{\mu} \circ \mathrm{st}^{-1}\right| \mathscr{B} \Leftrightarrow \mu \mid \mathscr{B} \text { is } \tau \text {-smooth. }
$$

Thus the above representation even characterizes the class of $\tau$-smooth Borel-measures.

If $\mu \mid \mathscr{B}$ is only a regular Borel-measure, it turns out that ${ }^{*} \bar{\mu} \circ \mathrm{st}^{-1} \mid \mathscr{B}$ is the greatest $\tau$-smooth minorant of $\mu \mid \mathscr{B}$ (Theorem 9(i)). This leads to a decomposition of the form

$$
\mu=\mu_{R}+\mu_{\tau}+\mu_{\sigma}
$$


where $\mu_{R}$ is Radon, $\mu_{\tau}$ is purely $\tau$-smooth, and $\mu_{\sigma}$ is purely $\sigma$-additive (for a standard proof of the result see [13]).

An essential tool for our considerations is the measurability of ns $\left({ }^{*} X\right)$ and of the standard part map st:ns $\left({ }^{*} X\right) \rightarrow X$. It turns out that st is universally Loeb-measurable on $\mathrm{ns}\left({ }^{*} X\right)$, i.e.

(3)

$$
\mathrm{st}^{-1}(B) \in L_{u}(* \mathscr{B}) \cap \mathrm{ns}\left({ }^{*} X\right)=\left\{C \cap \mathrm{ns}\left({ }^{*} X\right): C \in L_{u}(* \mathscr{B})\right\} \quad \text { for all } B \in \mathscr{B} .
$$

Hence st is universally Loeb-measurable iff $n s\left({ }^{*} X\right) \in L_{u}\left({ }^{*} \mathscr{B}\right)$ and therefore universally Loeb-measurable for many important topological spaces (see Corollary 3 ).

For applications in standard measure theory a stronger measurability of st than given in (3) is needed, namely

$$
\mathrm{st}^{-1}(B) \in L_{u}\left(* \mathscr{B}_{0}\right) \cap \mathrm{ns}(* X) \text { for all } B \in \mathscr{B},
$$

where $\mathscr{B}_{0}$ is the $\sigma$-algebra of Baire-sets of $X$, i.e. $\mathscr{B}_{0}$ is the smallest $\sigma$-algebra rendering all continuous real-valued functions measurable.

We show that (4) holds for each completely regular Hausdorff-space (Theorem 5). This yields two results of classical measure theory: The extension theorem for $\tau$-smooth Baire-measures to $\tau$-smooth Borel-measures and the theorem of Kakutani for product measures (Corollaries 13 and 14). Moreover we obtain representation and decomposition results for Baire-measures.

Basic for our investigations are Theorems 1 and 2 which show that unions and intersections of less than $K$-many sets of $* \mathscr{A}$ are universally Loeb-measurable and that each finite internal content $\left.\nu\right|^{*} \mathscr{A}$ is " $K$-smooth." A finite Borel-measure $\mu \mid \mathscr{B}$ is called regular iff $\mu(B)=\sup \{\mu(C): C \subset B, C$ closed $\}$ for all $B \in \mathscr{B}$; it is called Radon iff $\mu(B)=\sup \{\mu(K): K \subset B, K$ compact $\}$ for all $B \in \mathscr{B}$, and it is called $\tau$-smooth iff $\mu(T)=\sup _{S \in \mathscr{S}} \mu(S)$ for each system $\mathscr{S} \subset \mathscr{T}$ with $\mathscr{S} \uparrow T(\mathscr{S} \uparrow T$ means $\cup_{S \in \mathscr{S}} S=T$, and for all $S_{1}, S_{2} \in \mathscr{S}$ we have $S_{1} \cup S_{2} \subset S_{3}$ for some $\left.S_{3} \in \mathscr{S}\right)$. It is known that each Radon-measure is $\tau$-smooth. Denote by $\mathscr{T}_{0}=$ $\left\{f^{-1}(\mathcal{O}): \mathcal{O} \subset \mathbf{R}\right.$ open, $f$ continuous $\}$ the system of exact open sets of $X$. The complements of the exact open sets are the exact closed sets.

A finite Baire-measure $\mu \mid \mathscr{B}_{0}$ is called Radon iff for every $\varepsilon>0$ there exists a compact set $K$ such that $\mu(B)<\varepsilon$ for all Baire-sets $B \subset X-K$; it is called $\tau$-smooth iff $\mu(T)=\sup _{S \in \mathscr{S}} \mu(S)$ for each $\mathscr{S} \subset \mathscr{T}_{0}$ with $\mathscr{S} \uparrow T \in \mathscr{T}_{0}$.

Let us remark that each finite Baire-measure $\mu \mid \mathscr{B}_{0}$ is regular, i.e. $\mu(B)=$ $\sup \{\mu(C): C \subset B, C$ exact closed $\}$ for all $B \in \mathscr{B}_{0}$.

2. Universal Loeb-measurability of sets and of the standard part map. In this section we prove results which hold for all finite internal contents, postponing the results for internal contents of the form ${ }^{*} \mu$ to $\S 3$. Results for internal contents are useful in all cases in which nonstandard theory is applied to families of classical measures: If $\mathscr{M}$ is a family of measures, then ${ }^{*} \mathscr{M}$ contains internal contents which are not of the form ${ }^{*} \mu$.

We recall that we consider $K$-saturated nonstandard models. 
1. Theorem. Let $\mathscr{A}$ be an algebra on $X$ and $\mathscr{S} \subset{ }^{*} \mathscr{A}$ be a subsystem with card $\mathscr{S}<K$. Then the following assertions hold.

(i) $\cup_{S \in \mathscr{S}} S$ and $\bigcap_{S \in \mathscr{S}} S$ are universally Loeb-measurable;

(ii) $\nu_{L}(T)=\sup _{S \in \mathscr{S}} \nu_{L}(S)\left[\nu_{L}(T)=\inf _{S \in \mathscr{S}} \nu_{L}(S)\right]$ if $\mathscr{S} \uparrow T[\mathscr{S} \downarrow T]$ and $\left.\nu\right|^{*} \mathscr{A}$ is a finite internal content.

Proof. (i) Let $\left.\nu\right|^{*} \mathscr{A}$ be a finite internal content. Put $V:=\bigcup_{S \in \mathscr{S}} S$. We have to show

$$
\alpha:=\sup \left\{\nu_{L}(A): * \mathscr{A} \ni A \subset V\right\}=\inf \left\{\nu_{L}(B): V \subset B \in * \mathscr{A}\right\}=: \beta .
$$

Let $\varepsilon>0$ be arbitrary but fixed, and put for $S \in \mathscr{S}$

$$
\mathscr{F}_{S}:=\left\{A \in \mathcal{A}^{*}: S \subset A, \nu(A) \leqslant \alpha+\varepsilon\right\} .
$$

Then $\left\{\mathscr{F}_{S}: S \in \mathscr{S}\right\}$ is a family of internal sets with the finite intersection property. As card $\mathscr{S}<K$ and as our model is $K$-saturated, there exists $B \in \bigcap_{S \in \mathscr{S}} \mathscr{F}_{S}$. Then $V \subset B \in *^{*}$ and $\nu_{L}(B) \leqslant \alpha+\varepsilon$. Hence $\beta \leqslant \alpha+\varepsilon$, whence $\beta \leqslant \alpha$. As obviously $\alpha \leqslant \beta$, we have $\alpha=\beta$. Thus $\bigcup_{S \in \mathscr{S}} S$ is universally Loeb-measurable. The assertion for $\bigcap_{S \in \mathscr{S}} S$ follows by forming complements.

(ii) We only consider $\mathscr{S} \uparrow T$. For each $\varepsilon>0$ there exists by (i) a set $A \in \in^{*} \mathscr{A}$ with $A \subset T$ and $\nu_{L}(A) \geqslant \nu_{L}(T)-\varepsilon$. As $A$ and all $S \in \mathscr{S}$ are internal and card $\mathscr{S}<K$, we obtain by $K$-saturation a finite subsystem $\mathscr{S}_{0} \subset \mathscr{S}$ with $A \subset \cup_{S \in \mathscr{S}_{0}} S$. As $\mathscr{S} \uparrow T$, there exists $S \in \mathscr{S}$ with $A \subset S$. Hence $\nu_{L}(S) \geqslant \nu_{L}(T)-\varepsilon$. This proves (ii).

The following theorem shows that the first part of Theorem 1(ii) can be generalized. This result will be used in Theorem 4(ii).

2. TheOREM. Let $\mathscr{A}$ be an algebra on $X$ and $\mathscr{S} \subset * \mathscr{A}$ be a subsystem with card $\mathscr{S}<K$. Let $\left.\nu\right|^{*} \mathscr{A}$ be a finite internal content. Then for every subset $Z \subset{ }^{*} X$ the following assertions hold.

(i) $\mathscr{S} \uparrow T \Rightarrow \bar{\nu}(T \cap Z)=\sup _{S \in \mathscr{S}} \bar{\nu}(S \cap Z)$;

(ii) $\mathscr{S} \downarrow T \Rightarrow \bar{\nu}(T \cap Z)=\inf _{S \in \mathscr{S}} \bar{\nu}(S \cap Z)$.

Proof. (i) Let $\varepsilon>0$ be given. By definition of $\bar{\nu}$ there exists $Z_{\varepsilon} \in *^{*}$ with $Z \subset Z_{\varepsilon}$ and $\bar{\nu}\left(Z_{\varepsilon}\right) \leqslant \bar{\nu}(Z)+\varepsilon$.

This implies for every $A \in L(\nu, * \mathscr{A})$ that

$$
\bar{\nu}\left(A \cap Z_{\varepsilon}\right) \leqslant \bar{\nu}(A \cap Z)+\varepsilon
$$

(as $Z \subset Z_{\varepsilon}$ and $A, Z_{\varepsilon}$ are $\nu_{L}$-measurable, we have, using the Carathéodory-measurability criterion,

$$
\begin{aligned}
\bar{\nu}\left(A \cap Z_{\varepsilon}\right) & +\bar{\nu}(\bar{A} \cap Z)-\varepsilon \leqslant \bar{\nu}\left(A \cap Z_{\varepsilon}\right)+\bar{\nu}\left(\bar{A} \cap Z_{\varepsilon}\right)-\varepsilon \\
= & \left.\bar{\nu}\left(Z_{\varepsilon}\right)-\varepsilon \leqslant \bar{\nu}(Z) \leqslant \bar{\nu}(A \cap Z)+\bar{\nu}(\bar{A} \cap Z)\right) .
\end{aligned}
$$

We have $S \cap Z_{\varepsilon} \uparrow T \cap Z_{\varepsilon}$ with $S \cap Z_{\varepsilon} \in{ }^{*} \mathscr{A}$ for $S \in \mathscr{S}$. Hence Theorem 1(ii) and (1) imply

$$
\begin{aligned}
\sup _{S \in \mathscr{S}^{\mathcal{D}}} \bar{\nu}(S \cap Z) & \geqslant \sup _{\text {(1) }} \bar{\nu}\left(S \cap \mathscr{S}^{\circ}\left(S Z_{\varepsilon}\right)-\varepsilon=\bar{\nu}\left(T \cap Z_{\varepsilon}\right)-\varepsilon\right. \\
& \geqslant \bar{\nu}(T \cap Z)-\varepsilon .
\end{aligned}
$$

(ii) follows by similar methods. 
Let us remark that-by the same method-Theorems 1 and 2 can be proven for finite internal contents on an internal algebra.

Important external sets in nonstandard topology, besides the set $n s\left({ }^{*} X\right)$ of near-standard points, are the set pns $\left({ }^{*} X\right)$ of pre-near-standard points and the set $\operatorname{cpt}\left({ }^{*} X\right)$ of compact points, which are used e.g. to characterize locally compact spaces, respectively complete spaces $($ see $[12,14])$. We show that in many cases these sets are universally Loeb-measurable.

The sets $\operatorname{cpt}\left({ }^{*} X\right)$ and $\operatorname{pns}\left({ }^{*} X\right)$ are defined by

$$
\begin{gathered}
\operatorname{cpt}\left({ }^{*} X\right)=\bigcup\left\{{ }^{*} K: K \subset X, K \text { compact }\right\} ; \\
\operatorname{pns}\left({ }^{*} X\right)=\left\{x \in{ }^{*} X \mid y \in m(x) \Rightarrow^{\circ}\left({ }^{*} \delta(x, y)\right)=0\right\},
\end{gathered}
$$

where for the definition of $\operatorname{pns}\left({ }^{*} X\right)$ we assume that $X$ is metric with distance function $\delta$; the monad $m(x)$ is defined for all $x \in{ }^{*} X$ by $m(x)=\bigcap\left\{{ }^{*} T: x \in{ }^{*} T, T\right.$ open\}.

3. Corollary. Let $(X, \mathscr{T})$ be a Hausdorff-space with Borel-algebra $\mathscr{B}$. Then:

(i) $\operatorname{cpt}\left({ }^{*} X\right) \in L_{u}\left({ }^{*} \mathscr{B}\right)$;

(ii) pns $\left({ }^{*} X\right) \in L_{u}(* \mathscr{B})$ for metric spaces;

(iii) $\mathrm{ns}\left({ }^{*} X\right) \in L_{u}\left({ }^{*} \mathscr{B}\right)$ for locally compact spaces, for $\sigma$-compact spaces, and for complete metric spaces;

(iv) $\mathrm{st}^{-1}(B) \in L_{u}\left({ }^{*} \mathscr{B}\right) \cap \mathrm{ns}\left({ }^{*} X\right), B \in \mathscr{B}$, for regular spaces.

Proof. Theorem 1 will be applied to $\mathscr{A}:=\mathscr{B}$.

(i) Put $\mathscr{S}:=\left\{{ }^{*} K: X \supset K\right.$ compact $\}$. Then card $\mathscr{S} \leqslant$ card $\mathscr{T}<K$ and (i) follows from Theorem 1(i).

(ii) Part (ii) is shown by Theorem 1 if we prove (with $K_{\varepsilon}(x)=\{y \in X: \delta(x, y)<$ $\varepsilon\})$

$$
\operatorname{pns}\left({ }^{*} X\right)=\bigcap_{n \in \mathbf{N}} \bigcup_{x \in X}{ }^{*} K_{1 / n}(x) .
$$

" $\supset$ " follows directly. For the converse direction let $y \in \operatorname{pns}\left({ }^{*} X\right)$. Then for all $n \in \mathbf{N}: m(y) \subset\left\{x \in{ }^{*} X:{ }^{*} \delta(x, y)<1 / n\right\}$. As $m(y)=\bigcap\left\{{ }^{*} T: y \in{ }^{*} T, T \in \mathscr{T}\right\}$, and as our model is $K$-saturated with $K>$ card $\mathscr{T}$, there exists $T\left(=T_{n}\right) \in \mathscr{T}$ such that

$$
m(y) \subset{ }^{*} T \subset\left\{x \in{ }^{*} X:{ }^{*} \delta(x, y)<1 / n\right\} .
$$

Choose $x_{n} \in T$; then ${ }^{*} \delta\left(x_{n}, y\right)<1 / n$. Hence $y \in{ }^{*} K_{1 / n}\left(x_{n}\right)$.

(iii) Since $\mathrm{ns}\left({ }^{*} X\right)=\operatorname{cpt}\left({ }^{*} X\right)$ for a locally compact space (see Theorem 8.3.14 of [14]) and $\mathrm{ns}\left({ }^{*} X\right)=\operatorname{pns}\left({ }^{*} X\right)$ for a complete space (see Theorem 3.14 .1 of [12]), it remains to consider the $\sigma$-compact case. Let $X=\bigcup_{n \in \mathrm{N}} K_{n}$ with compact $K_{n}$. Using Lemma 3.4 .4 of [1], we obtain

$$
\mathrm{ns}\left({ }^{*} X\right)=\mathrm{st}^{-1}(X)=\bigcup_{n \in \mathbf{N}} \mathrm{st}^{-1}\left(K_{n}\right)=\bigcup_{n \in \mathbb{N}} \bigcap\left\{{ }^{*} T: K_{n} \subset T, T \text { open }\right\},
$$

and hence ns $\left({ }^{*} X\right) \in L_{u}\left({ }^{*} \mathscr{B}\right)$ according to Theorem 1(i). 
(iv) Let $C \subset X$ be closed. Put $m(C):=\bigcap\left\{{ }^{*} T: C \subset T \in \mathscr{T}\right\}$. Then $m(C) \in$ $L_{u}(* \mathscr{B})$ by Theorem 1(i). Therefore it suffices to prove $\mathrm{st}^{-1}(C)=m(C) \cap \mathrm{ns}\left({ }^{*} X\right)$ for a regular space: the inclusion " $\subset$ " is obvious; for " $\supset$ " let $y \in m(C) \cap \mathrm{ns}(* X)$. Then there exists $x \in X$ with $y \in m(x)$. If $x \notin C$ then by regularity of $X$ there would exist disjoint open sets $T_{1}, T_{2}$ with $x \in T_{1}, C \subset T_{2}$. Hence $m(C) \cap m(x) \subset{ }^{*} T_{1} \cap * T_{2}=\varnothing$, yielding a contradiction.

For locally compact spaces (see [1, 11]) and complete metric spaces (see [11]) part (iii) of Corollary 3 is known.

Since by Corollary 3(iv) the measurability of st is equivalent to the measurability of $\mathrm{ns}\left({ }^{*} X\right)$, it is of interest to obtain $\mathrm{ns}\left({ }^{*} X\right) \in L_{u}\left({ }^{*} \mathscr{B}\right)$ for more general topological spaces than in 3(iii). However, if $X$ is a separable metric space or a complete uniform space, then $n s\left({ }^{*} X\right)$ need not be universally Loeb-measurable.

(a) There exist separable metric spaces $X$ which admit a non-Radon Borel-measure, say $\mu$. Then $\mu$ is $\tau$-smooth and therefore $n s\left({ }^{*} X\right) \notin L\left({ }^{*} \mu,{ }^{*} \mathscr{B}\right)$ by Corollary 11 . Hence $\mathrm{ns}\left({ }^{*} X\right) \notin L_{u}(* \mathscr{B})$.

(b) $X=\mathbf{R}^{\mathbf{R}}$ is a complete uniform space which admits a $\tau$-smooth Borel-measure which is non-Radon. Then $\mathrm{ns}\left({ }^{*} X\right) \notin L_{u}(* \mathscr{B})$ by Corollary 11 .

As in complete uniform spaces pns $\left({ }^{*} X\right)=\mathrm{ns}\left({ }^{*} X\right)$ (see Theorem 3.14.1 of [12]), part (b) above shows that pns $\left({ }^{*} X\right) \notin L_{u}(* \mathscr{B})$ for certain complete uniform spaces.

As the measurability of the standard part map on $n s\left({ }^{*} X\right)$ is fundamental for this paper, let us remark that Corollary 3(iv) does not hold without the assumption that $(X, \mathscr{T})$ is regular: According to Example 15, even for $\tau$-smooth measures $\mu$ in a Hausdorff-space, it may happen that $\mathrm{st}^{-1}(B) \notin L\left({ }^{*} \mu,{ }^{*} \mathscr{B}\right) \cap \mathrm{ns}\left({ }^{*} X\right)$ for some $B \in$ $\mathscr{B}$. If, however, $\mu$ is a regular measure in a Hausdorff-space, we obtain $\operatorname{st}^{-1}(B) \in$ $L\left({ }^{*} \mu,{ }^{*} \mathscr{B}\right) \cap \mathrm{ns}\left({ }^{*} X\right)$ for all $B \in \mathscr{B}$ (see Lemma $8(\mathrm{i})$ ).

In the following theorem we prove in (i) a stronger form of measurability of the standard part map than given in Corollary 3(iv). Such a stronger form of measurability is useful for proving extension theorems of measures.

4. TheOREM. Let $(X, \mathscr{T})$ be a regular Hausdorff-space, let $\mathscr{T}_{1}$ be a base of $\mathscr{T}$, and let $\mathscr{B}_{1}$ be the $\sigma$-algebra generated by $\mathscr{T}_{1}$.

(i) If $B$ is a Borel-subset of $X$ then $\mathrm{st}^{-1}(B) \in L_{u}\left(* \mathscr{B}_{1}\right) \cap \mathrm{ns}(* X)$.

(ii) If $\left.\nu\right|^{* \mathscr{B}_{1}}$ is a finite internal content, then $\bar{\nu} \circ \mathrm{st}^{-1} \mid \mathscr{B}$ is a $\tau$-smooth measure and $\underline{\nu} \circ \mathrm{st}^{-1} \mid \mathscr{B}$ is a Radon-measure.

Proof. (i) Let $\left.\nu\right|^{*} \mathscr{B}_{1}$ be a finite internal content. It suffices to prove that for every closed set $C \subset X$

$$
\mathrm{st}^{-1}(C) \in L\left(\nu, \mathscr{B}_{1}\right) \cap \mathrm{ns}(* X) .
$$

We shall show that for every open set $T \supset C$ there exists $B_{T} \in *^{*} \mathscr{B}_{1}$ with

$$
\mathrm{st}^{-1}(C) \subset B_{T} \subset{ }^{*} T \text {. }
$$

Let us at first show that (2) implies (1). Let $m(C)=\bigcap\left\{{ }^{*} T: C \subset T \in \mathscr{T}\right\}$. Then (2) implies

$$
\mathrm{st}^{-1}(C) \subset \cap\left\{B_{T}: C \subset T \in \mathscr{T}\right\} \subset m(C) .
$$


As our model is $K$-saturated with $\operatorname{card}(\mathscr{T})<K$, we obtain by Theorem 1(i)-applied to $\mathscr{A}=\mathscr{B}_{1}-$ that $B:=\bigcap\left\{B_{T}: C \subset T \in \mathscr{T}\right\} \in L\left(\nu, \mathscr{B}_{1}\right)$. Hence by (3)

$$
\mathrm{st}^{-1}(C) \subset B \subset m(C) \text { for some } B \in L\left(\nu, \mathscr{B}_{1}\right) .
$$

As $(X, \mathscr{T})$ is regular, and $C$ is closed, we have

$$
\mathrm{st}^{-1}(C)=m(C) \cap \mathrm{ns}\left({ }^{*} X\right) .
$$

Hence we obtain $\mathrm{st}^{-1}(C)=B \cap \mathrm{ns}\left({ }^{*} X\right)$ for some $B \in L\left(\nu,{ }^{*} \mathscr{B}_{1}\right)$, i.e. (1) holds.

It remains to prove (2). To this aim let $T \supset C$ be a fixed open set. For every $x \in C$ there exists $T_{x} \in \mathscr{T}_{1}$ with $x \in T_{x} \subset T$. Put for $x \in C$

$$
\mathscr{S}_{x}=\left\{B \in \mathscr{B}_{1}:{ }^{*} T_{x} \subset B \subset{ }^{*} T\right\} .
$$

Then $\mathscr{S}_{x}, x \in C$, is a system of internal sets. As $* \mathscr{B}_{1}$ is closed under finite unions, $\left\{\mathscr{S}_{x}: x \in C\right\}$ has the finite intersection property. As our model is $K$-saturated with $K>\operatorname{card}(C)$, we obtain $\bigcap_{x \in C} \mathscr{S}_{x} \neq \varnothing$. Let $B \in \bigcap_{x \in C} \mathscr{S}_{x}$. Then $B \in * \mathscr{B}_{1}$, and ${ }^{*} T_{x} \subset B \subset{ }^{*} T$ for all $x \in C$. Hence $m(x) \subset B \subset{ }^{*} T$ for all $x \in C$, whence $\mathrm{st}^{-1}(C)$ $=\bigcup_{x \in C} m(x) \subset B \subset{ }^{*} T$. This proves (2) and hence (i).

(ii) W.l.g. let $\mathscr{T}_{1}$ be closed under finite unions. As $\nu_{L}$ is a measure on $L\left(\nu, \mathscr{B}_{1}\right)$, we obtain that $\bar{\nu}$ and $\underline{\nu}$ are measures on $L\left(\nu, \mathscr{B}_{1}\right) \cap \mathrm{ns}\left({ }^{*} X\right)$. Hence by (i) we obtain that $\bar{\nu} \circ \mathrm{st}^{-1} \mid \mathscr{B}$ and $\underline{\nu} \circ \mathrm{st}^{-1} \mid \mathscr{B}$ are Borel-measures. We prove at first that $\bar{\nu} \circ \mathrm{st}^{-1} \mid \mathscr{B}$ is $\tau$-smooth. Let $\mathscr{T} \supset \mathcal{O} \uparrow T$. We have to prove

$$
\bar{\nu}\left(\mathrm{st}^{-1}(T)\right) \leqslant \sup _{U \in \mathcal{O}} \bar{\nu}\left(\mathrm{st}^{-1}(U)\right) .
$$

As $\mathscr{T}_{1}$ is a base for the topology and $X$ is a regular space, we can choose for each pair $(x, U)$ with $x \in U \in \mathcal{O}$ a set $H_{x, U} \in \mathscr{T}_{1}$ with

$$
x \in H_{x, U} \subset \mathrm{cl} H_{x, U} \subset U,
$$

where cl $H$ denotes the closure of $H$.

Let $\mathscr{H}$ be the system of all finite unions of sets $H_{x, U}$ with $x \in U \in \mathcal{O}$. As $\mathcal{O}$ is directed upwards, we obtain by (5) that

$$
\forall H \in \mathscr{H} \exists U \in \mathcal{O} \text { with } H \subset \mathrm{cl} H \subset U .
$$

Put $\mathscr{S}=\left\{{ }^{*}(\mathrm{cl} H): H \in \mathscr{H}\right\}$. If $C \subset U, C$ closed, $U$ open, then $\mathrm{ns}\left({ }^{*} X\right) \cap{ }^{*} C \subset$ $\mathrm{st}^{-1}(U)$. Hence relation (6) implies

$$
\forall S \in \mathscr{S} \exists U \in \mathcal{O} \text { with } n s\left({ }^{*} X\right) \cap S \subset \mathrm{st}^{-1}(U) .
$$

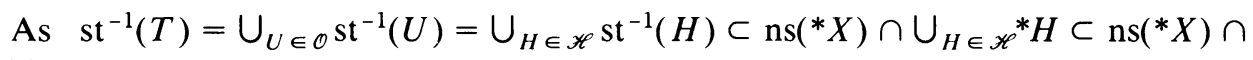
$\cup_{S \in \mathscr{S}} S$ by (5) and (6), we obtain by (7) that

$$
\mathrm{st}^{-1}(T)=\mathrm{ns}(* X) \cap \bigcup_{S \in \mathscr{S}} S .
$$

To apply Theorem 2 put $\mathscr{A}=\mathscr{B}_{1}$ and $Z=\mathrm{ns}\left({ }^{*} X\right)$. As our model is $K$-saturated with $K>\operatorname{card}(\mathscr{T})$, we obtain by (8), Theorem 2 , and (7)

$$
\begin{aligned}
\bar{\nu}\left(\mathrm{st}^{-1}(T)\right) & \underset{(8)}{=} \bar{\nu}\left(\mathrm{ns}\left({ }^{*} X\right) \cap \bigcup_{S \in \mathscr{S}} S\right) \underset{\text { Th.2 }}{=} \sup _{S \in \mathscr{S}} \bar{\nu}\left(\mathrm{ns}\left({ }^{*} X\right) \cap S\right) \\
& \leqslant \sup _{(7)} \bar{\nu}\left(\mathrm{st}^{-1}(U)\right) .
\end{aligned}
$$

This proves (4) and hence we have shown that $\bar{\nu} \circ \mathrm{st}^{-1} \mid \mathscr{B}$ is a $\tau$-smooth measure. 
For (ii) it remains to prove that $\underline{\nu} \circ \mathrm{st}^{-1} \mid \mathscr{B}$ is a Radon-measure: By definition of $\underline{\nu}$ we have for each $B \in \mathscr{B}$

$$
\begin{aligned}
\underline{\nu}\left(\mathrm{st}^{-1}(B)\right) & =\sup \left\{\nu_{L}(A): * \mathscr{B}_{1} \ni A \subset \mathrm{st}^{-1}(B)\right\} \\
& \leqslant \sup \left\{\underline{\nu}\left(\mathrm{st}^{-1}(\mathrm{st} A)\right): * \mathscr{B}_{1} \ni A \subset \mathrm{st}^{-1}(B)\right\} .
\end{aligned}
$$

Since $X$ is regular, we have that $\operatorname{st}(A)$ is compact for each internal $A \subset \mathrm{ns}\left({ }^{*} X\right)$. Hence

$$
\underline{\nu}\left(\mathrm{st}^{-1}(B)\right) \leqslant \sup \left\{\underline{\nu}\left(\mathrm{st}^{-1}(K)\right): K \subset B, K \text { compact }\right\},
$$

i.e. the assertion.

According to Example 15, all assertions of Theorem 4 are not true any more if we omit the regularity of $X$, even if $\mathscr{T}_{1}=\mathscr{T}$ and $\nu={ }^{*} \mu$ with $\tau$-smooth measure $\mu$.

The following result is a direct consequence of Theorem 4(i). If $X$ is compact, this result is due to Loeb (see Corollary 3 of [8]; Loeb also gives some extensions).

5. TheOREM. Let $(X, \mathscr{T})$ be a completely regular Hausdorff-space with Baire- $\sigma$ algebra $\mathscr{B}_{0}$. Then

$$
\mathrm{st}^{-1}(B) \in L_{u}\left(* \mathscr{B}_{0}\right) \cap \mathrm{ns}(* X) \quad \text { for each Borel-set } B \text { of } X .
$$

Proof. As $X$ is a completely regular Hausdorff-space, the system $\mathscr{T}_{0}$ of exact open sets is a base for the topology $\mathscr{T}$. Now the assertion follows from Theorem 4(i), applied to $\mathscr{T}_{1}=\mathscr{T}_{0}$ and $\mathscr{B}_{1}=\mathscr{B}_{0}$.

3. Representations of $\tau$-smooth Baire- and Borel-measures with applications. In this section we give results for measures ${ }^{*} \mu$, where $\mu$ is a standard measure. The following three lemmata are essential tools for proving our theorems.

If $\mathscr{S} \subset \mathscr{P}(X)$ then $\mathscr{S}^{U}$ denotes the system of all finite unions of elements of $\mathscr{S}$, and $\overline{\mathscr{S}}$ denotes the system of complements of elements of $\mathscr{S}$.

6. Lemma. Let $(X, \mathscr{T})$ be a Hausdorff-space. Let $\mathscr{T}_{1}$ be a base of $\mathscr{T}$ and $\mathscr{B}_{1}$ be the $\sigma$-algebra generated by $\mathscr{T}_{1}$. Then we have for each finite measure $\mu \mid \mathscr{B}_{1}$ :

(i) $\sup _{T \in \mathcal{O}} \mu(T)=\mu(X)$ for every $\mathcal{O} \subset \mathscr{T}_{1}^{U}$ with $\mathcal{O} \uparrow X \Leftrightarrow * \bar{\mu}\left(\mathrm{ns}\left({ }^{*} X\right)\right)=\mu(X)$;

(ii) if $*^{*}\left(\mathrm{~ns}\left({ }^{*} X\right)\right)=\mu(X)$ and $\mu\left(B_{1}\right)=\inf \left\{\mu\left(T_{1}\right): B_{1} \subset T_{1} \in \mathscr{T}_{1}\right\}$ for all $B_{1} \in$ $\mathscr{B}_{1}$ then $\mu\left|\mathscr{B}_{1}={ }^{*} \bar{\mu} \circ \mathrm{st}^{-1}\right| \mathscr{B}_{1}$.

Proof. (i) $\Rightarrow$ Let $B \in *^{*} \mathscr{B}_{1}$ with ns $\left.{ }^{*} X\right) \subset B$ be given. We have to prove

$$
{ }^{*} \mu_{L}(B)=\mu(X) \text {. }
$$

Let $x \in X$ be given. Then $m(x)=\bigcap_{x \in T \in \mathscr{T}_{1}}{ }^{*} T \subset B$, as $\mathscr{T}_{1}$ is a base for the topology and $\mathrm{ns}\left({ }^{*} X\right) \subset B$. As our model is $K$-saturated with $K>\operatorname{card}(\mathscr{T})$, there exists $T_{x} \in \mathscr{T}_{1}$ with $x \in T_{x},{ }^{*} T_{x} \subset B$. Hence we have for each finite $E \subset X$

$$
\begin{aligned}
\mu(X) & =*^{*} \mu_{L}(* X) \geqslant * \mu_{L}(B) \geqslant * \mu_{L}\left(\bigcup_{x \in E} * T_{x}\right) \\
& =* \mu_{I}\left(*\left(\bigcup_{x \in E} T_{x}\right)\right)=\mu\left(\bigcup_{x \in E} T_{x}\right) .
\end{aligned}
$$


As $T_{x} \in \mathscr{T}_{1}$, we have

$$
\mathscr{T}_{1}^{U} \supset\left\{\bigcup_{x \in E} T_{x}: E \subset X \text { finite }\right\} \uparrow X .
$$

Hence we obtain by assumption

$$
\mu(X)=\sup \left\{\mu\left(\bigcup_{x \in E} T_{x}\right): E \subset X \text { finite }\right\}
$$

Hence (2) implies (1).

(i) $\Leftarrow$ For the converse direction let $\mathcal{O} \subset \mathscr{T}_{1}^{U}$ with $\mathcal{O} \uparrow X$ be given. Then $\mathrm{ns}\left({ }^{*} X\right) \subset$ $\cup_{T \in \mathcal{O}}{ }^{*} T$. Now we obtain by assumption and Theorem 1(i),(ii)

$$
\mu(X)=*^{*} \bar{\mu}\left(\mathrm{ns}\left({ }^{*} X\right)\right) \leqslant{ }^{*} \mu_{L}\left(\bigcup_{T \in \mathcal{O}}{ }^{*} T\right)=\sup _{T \in \mathcal{O}}{ }^{*} \mu_{L}\left({ }^{*} T\right)=\sup _{T \in \mathcal{O}} \mu(T) .
$$

(ii) For each $B_{1} \in \mathscr{B}_{1}$ we have, as ${ }^{*} \bar{\mu}\left(\mathrm{ns}\left({ }^{*} X\right)\right)=\mu(X)$ and $\mu\left(B_{1}\right)=\sup \{\mu(C)$ : $\left.\overline{\mathscr{T}}_{1} \ni C \subset B_{1}\right\}$,

$$
\begin{aligned}
\mu\left(B_{1}\right) & =\sup \left\{{ }^{*} \mu\left({ }^{*} C\right): \overline{\mathscr{T}}_{1} \ni C \subset B_{1}\right\}=\sup \left\{{ }^{*} \bar{\mu}\left({ }^{*} C \cap \mathrm{ns}\left({ }^{*} X\right)\right): \overline{\mathscr{T}}_{1} \ni C \subset B_{1}\right\} \\
& \leqslant \sup \left\{{ }^{*} \bar{\mu}\left(\mathrm{st}^{-1}(C)\right): \overline{\mathscr{T}}_{1} \ni C \subset B_{1}\right\} \leqslant{ }^{*} \bar{\mu}\left(\mathrm{st}^{-1}\left(B_{1}\right)\right) \\
& \leqslant \inf \left\{{ }^{*} \bar{\mu}\left(\mathrm{st}^{-1}(0)\right): B_{1} \subset 0 \in \mathscr{T}_{1}\right\} \leqslant \inf \left\{\mu(0): B_{1} \subset 0 \in \mathscr{T}_{1}\right\}=\mu\left(B_{1}\right) .
\end{aligned}
$$

7. Lemma. Let $(X, \mathscr{T})$ be a Hausdorff-space with Borel-algebra $\mathscr{B}$. Let $\left.\nu\right|^{* \mathscr{B}}$ be a finite internal content. Then

$$
\bar{\nu}\left(\mathrm{st}^{-1}(E)\right)=\inf \left\{\bar{\nu}\left(\mathrm{st}^{-1}(T)\right): E \subset T \in \mathscr{T}\right\} \quad \text { for every subset } E \text { of } X .
$$

Proof. As $\bar{\nu} \mid \mathscr{P}\left({ }^{*} X\right)$ is monotone, it suffices to show

$$
\inf \left\{\bar{\nu}\left(\mathrm{st}^{-1}(T)\right): E \subset T \in \mathscr{T}\right\} \leqslant \bar{\nu}\left(\mathrm{st}^{-1}(E)\right) .
$$

By definition of $\bar{\nu}$ we have for every $E \subset X$,

$$
\bar{\nu}\left(\mathrm{st}^{-1}(E)\right)=\inf \left\{\nu_{L}(A): \mathrm{st}^{-1}(E) \subset A \in * \mathscr{B}\right\} .
$$

Now let $A \in * \mathscr{B}$ with $A \supset \mathrm{st}^{-1}(E)$ be given. Then $m(x)=\bigcap\left\{{ }^{*} 0: x \in 0 \in \mathscr{T}\right\} \subset A$ for each $x \in E$. As our model is $K$-saturated with $K>\operatorname{card}(\mathscr{T})$, there exist open sets $T_{x}$ with $x \in T_{x}$ and ${ }^{*} T_{x} \subset A$. Hence $T_{1}=\cup_{x \in E} T_{x}$ is an open set with $E \subset T_{1}$ and $\mathrm{st}^{-1}\left(T_{1}\right)=\cup_{x \in E} \mathrm{st}^{-1}\left(T_{x}\right) \subset \cup_{x \in E} T_{x} \subset A$. Hence

$$
\inf \left\{\bar{\nu}\left(\mathrm{st}^{-1}(T)\right): E \subset T \in \mathscr{T}\right\} \leqslant \bar{\nu}\left(\mathrm{st}^{-1}\left(T_{1}\right)\right) \leqslant \nu_{L}(A) .
$$

According to (2), this implies (1), i.e. the assertion.

8. Lemma. Let $(X, \mathscr{T})$ be a Hausdorff-space with Borel-algebra $\mathscr{B}$ and $\mu \mid \mathscr{B}$ be a finite regular Borel-measure. Then

(i) $\mathrm{st}^{-1}(B) \in L(* \mu, * \mathscr{B}) \cap \mathrm{ns}\left({ }^{*} X\right)$ for all $B \in \mathscr{B}$;

(ii) ${ }^{*} \bar{\mu} \circ \mathrm{st}^{-1} \mid \mathscr{B}$ is a regular $\tau$-smooth minorant of $\mu \mid \mathscr{B}$.

Proof. (i) We have for each closed set $C$ that

$$
{ }^{*} C \cap \mathrm{ns}(* X) \subset \mathrm{st}^{-1}(C) \subset m(C) \cap \mathrm{ns}\left({ }^{*} X\right) \text {. }
$$

From (1) we obtain $\mathrm{st}^{-1}(C)=D \cap \mathrm{ns}\left({ }^{*} X\right)$ for some ${ }^{*} C \subset D \subset m(C)$. Furthermore, as $\mu$ is regular, ${ }^{*} \mu_{L}\left({ }^{*} C\right)={ }^{*} \mu_{L}(m(C))$ and hence $D \in L\left({ }^{*} \mu,{ }^{*} \mathscr{B}\right)$. 
(ii) According to (i) and Lemma $7, *^{*} \circ s t^{-1} \mid \mathscr{B}$ is a regular measure. It suffices to prove

$$
\mathscr{T} \ni T_{\alpha} \uparrow X \Rightarrow{ }^{*} \bar{\mu} \circ \mathrm{st}^{-1}\left(T_{\alpha}\right) \uparrow * \bar{\mu} \circ \mathrm{st}^{-1}(X) .
$$

According to Theorem 2, it suffices to prove

$$
{ }^{*} \bar{\mu} \circ \mathrm{st}^{-1}(T)={ }^{*} \bar{\mu}\left({ }^{*} T \cap \mathrm{ns}(* X)\right) .
$$

Using ${ }^{*} \mu_{I .}\left({ }^{*} C\right)={ }^{*} \mu_{I}(m(C))$ for each closed set and using ${ }^{*} C, m(C) \in L\left({ }^{*} \mu,{ }^{*} \mathscr{B}\right)$ we obtain that

$$
{ }^{*} \bar{\mu}\left({ }^{*} C \cap \mathrm{ns}\left({ }^{*} X\right)\right)=* \bar{\mu}(m(C) \cap \mathrm{ns}(* X)),
$$

and hence by (1)

$$
{ }^{*} \bar{\mu}\left({ }^{*} C \cap \mathrm{ns}(* X)\right)={ }^{*} \bar{\mu} \circ \mathrm{st}^{-1}(C) .
$$

Let $T$ be open and $C:=X-T$. Then

$$
\begin{aligned}
{ }^{*} \bar{\mu} \circ \mathrm{st}^{-1}(T) & ={ }^{*} \bar{\mu} \circ \mathrm{st}^{-1}(X-C)={ }^{*} \bar{\mu} \circ \mathrm{st}^{-1}(X)-{ }^{*} \bar{\mu} \circ \mathrm{st}^{-1}(C) \\
& ={ }^{*} \bar{\mu}\left(\mathrm{ns}\left({ }^{*} X\right)\right)-{ }^{*} \bar{\mu}\left({ }^{*} C \cap \mathrm{ns}\left({ }^{*} X\right)\right) \\
& (2) \\
& ={ }^{*} \bar{\mu}\left(\mathrm{ns}\left({ }^{*} X\right) \cap\left({ }^{*} X-{ }^{*} C\right)\right)={ }^{*} \bar{\mu}\left({ }^{*} T \cap \mathrm{ns}\left({ }^{*} X\right)\right) .
\end{aligned}
$$

Since $\mu \mid \mathscr{B}$ is regular, we obtain ${ }^{*} \bar{\mu} \circ \mathrm{st}^{-1}|\mathscr{B} \leqslant \mu| \mathscr{B}$ from the fact that ${ }^{*} \bar{\mu}\left(\mathrm{st}^{-1}(T)\right)$ $\leqslant{ }^{*} \bar{\mu}\left({ }^{*} T\right)=\mu(T)$ for each $T \in \mathscr{T}$.

Observe that the following theorem holds for Hausdorff-spaces and not only for regular Hausdorff-spaces. For regular Hausdorff-spaces parts of Theorem 9 can be deduced more directly by using Theorem 4 .

9. Theorem. Let $(X, \mathscr{T})$ be a Hausdorff-space with Borel-algebra $\mathscr{B}$. Let $\mu \mid \mathscr{B}$ be a finite Borel-measure. Then

(i) $\mu \mid \mathscr{B}$ regular $\Rightarrow{ }^{*} \bar{\mu} \circ \mathrm{st}^{-1} \mid \mathscr{B}$ is the greatest regular $\tau$-smooth minorant of $\mu \mid \mathscr{B}$;

(ii) $\mu \mid \mathscr{B}$ regular and $\tau$-smooth $\Leftrightarrow \mu\left|\mathscr{B}=* \bar{\mu} \circ \mathrm{st}^{-1}\right| \mathscr{B}$.

Proof. (ii) $\Rightarrow$ Apply Lemma 6(i) and (ii) to $\mathscr{T}_{1}=\mathscr{T}, \mathscr{B}_{1}=\mathscr{B}$.

(ii) $\Leftarrow \mu$ is regular according to Lemma 7 applied to $\nu=* \mu$. As $\mu(X)=$ $* \bar{\mu}\left(\mathrm{st}^{-1}(X)\right)={ }^{*} \bar{\mu}\left(\mathrm{ns}\left({ }^{*} X\right)\right), \mu$ is $\tau$-smooth in $X$ according to Lemma 6(i) and hence $\tau$-smooth as $\mu \mid \mathscr{B}$ is regular.

(i) According to Lemma $8,{ }^{*} \bar{\mu} \circ \mathrm{st}^{-1} \mid \mathscr{B}$ is a regular $\tau$-smooth minorant of $\mu \mid \mathscr{B}$. Let $\nu|\mathscr{B} \leqslant \mu| \mathscr{B}$ be regular and $\tau$-smooth. Then ${ }^{*} \bar{\nu} \circ \mathrm{st}^{-1}\left|\mathscr{B} \leqslant{ }^{*} \bar{\mu} \circ \mathrm{st}^{-1}\right| \mathscr{B}$. The assertion follows, as $\nu\left|\mathscr{B}=* \bar{\nu} \circ \mathrm{st}^{-1}\right| \mathscr{B}$ by (ii).

Let us remark that if $\mu \mid \mathscr{B}$ is only a $\tau$-smooth measure, then it cannot even be guaranteed that $* \bar{\mu} \circ \mathrm{st}^{-1} \mid \mathscr{B}$ is a measure (see Example 15 ).

Part "(ii) $\Rightarrow$ " of the next theorem is due to Anderson [2].

10. Proposition. Let $(X, \mathscr{T})$ be a regular Hausdorff-space with Borel-algebra $\mathscr{B}$. Let $\mu \mid \mathscr{B}$ be a finite Borel-measure. Then

(i) $\mu \mid \mathscr{B}$ regular $\Rightarrow{ }^{*} \mu \circ \mathrm{st}^{-1} \mid \mathscr{B}$ is the greatest Radon-minorant of $\mu \mid \mathscr{B}$;

(ii) $\mu \mid \mathscr{B}$ Radon $\Leftrightarrow \mu\left|\mathscr{B}={ }^{*} \mu \circ \mathrm{st}^{-1}\right| \mathscr{B}$. 
Proof. (ii) $\Leftarrow$ follows from Theorem 4(ii) applied to $\mathscr{T}_{1}=\mathscr{T}, \mathscr{B}_{1}=\mathscr{B}$, and $\nu=* \mu$.

The other direction holds even for Hausdorff-spaces (see Theorem 3.3 of [2]).

(i) According to Theorem 4(ii), we have that ${ }^{*} \mu \circ \mathrm{st}^{-1} \mid \mathscr{B}$ is Radon. As $\mu$ is regular, we have according to Theorem 1(ii) that

$$
{ }^{*} \underline{\mu}\left(\mathrm{st}^{-1}(K)\right)={ }^{*} \mu_{L}(m(K)) \underset{1(\mathrm{ii})}{=} \inf \{\mu(0): K \subset 0 \in \mathscr{T}\}=\mu(K)
$$

for all compact sets $K$. Hence ${ }^{*} \mu \circ \mathrm{st}^{-1} \mid \mathscr{B}$ is a Radon-minorant of $\mu \mid \mathscr{B}$. Let $\nu|\mathscr{B} \leqslant \mu| \mathscr{B}$ be Radon. Then ${ }^{*} \underline{\nu} \circ \mathrm{st}^{-1}\left|\mathscr{B} \leqslant{ }^{*} \mu \circ \mathrm{st}^{-1}\right| \mathscr{B}$, and hence the assertion follows as $\nu\left|\mathscr{B}={ }^{*} \underline{\nu} \circ \mathrm{st}^{-1}\right| \mathscr{B}$ by (ii).

From Theorem 9 and Proposition 10 we directly obtain for regular Borel-measures $\mu \mid \mathscr{B}$ in regular Hausdorff-spaces, that there exists a (unique) decomposition

$$
\mu=\mu_{R}+\mu_{\tau}+\mu_{\sigma},
$$

where $\mu_{R} \mid \mathscr{B}$ is Radon, $\mu_{\tau} \mid \mathscr{B}$ is purely $\tau$-smooth (i.e., $\mu_{\tau} \mid \mathscr{B}$ is $\tau$-smooth and $\nu\left|\mathscr{B} \leqslant \mu_{\tau}\right| \mathscr{B}$ with $\nu \mid \mathscr{B}$ Radon $\Rightarrow \nu \equiv 0$ ), and $\mu_{\sigma} \mid \mathscr{B}$ is purely $\sigma$-additive (i.e. $\mu_{\sigma} \mid \mathscr{B}$ is a measure and $\nu\left|\mathscr{B} \leqslant \mu_{\sigma}\right| \mathscr{B}$ with $\nu \mid \mathscr{B} \tau$-smooth $\left.\Rightarrow \nu \equiv 0\right)$. Put $\mu_{R}={ }^{*} \mu \circ \mathrm{st}^{-1}$, $\mu_{\tau}=*_{\bar{\mu}} \circ \mathrm{st}^{-1}-{ }^{*} \underline{\mu} \circ \mathrm{st}^{-1}$, and $\mu_{\sigma}=\mu-\mu_{R}-\mu_{\tau}$. Observe that $\mu_{R}(K)={ }^{*} \mu \circ \overline{s t}^{-1}(K)$ for all compact sets.

The standard proof for this decomposition theorem for regular Borel-measures is given in Saponakis and Sion [13].

11. Corollary. Let $(X, \mathscr{T})$ be a regular Hausdorff-space with Borel-algebra $\mathscr{B}$. Let $\mu \mid \mathscr{B}$ be a finite Borel-measure. Then

$$
\mu \mid \mathscr{B} \text { Radon } \Leftrightarrow \mu \mid \mathscr{B} \tau \text {-smooth and } \mathrm{ns}(* X) \in L\left({ }^{*} \mu,{ }^{*} \mathscr{B}\right) \text {. }
$$

Proof. Since $\mu \mid \mathscr{B}$ Radon implies $\mu \mid \mathscr{B} \quad \tau$-smooth and $\mu(X)={ }^{*} \mu\left(\mathrm{ns}\left({ }^{*} X\right)\right)$ by Proposition 10(ii), it remains to prove " $\Leftarrow "$ ": As in a regular Hausdorff-space a $\tau$-smooth Borel-measure is regular; we obtain by Theorem 9(ii) $\mu\left|\mathscr{B}={ }^{*} \bar{\mu} \circ \mathrm{st}^{-1}\right| \mathscr{B}$. Using Corollary 3 (iv) and $\mathrm{ns}\left({ }^{*} X\right) \in L\left({ }^{*} \mu,{ }^{*} \mathscr{B}\right)$ we obtain ${ }^{*} \bar{\mu} \circ \mathrm{st}^{-1}\left|\mathscr{B}={ }^{*} \mu \circ \mathrm{st}^{-1}\right| \mathscr{B}$. Hence Proposition 10(ii) implies the assertion.

Let us remark that Corollary 11 " $\Leftarrow "$ is not true without the assumption that $X$ is regular (use Example 15 and observe that ${ }^{*}{ }^{\circ} \mathrm{st}^{-1}(X)=1$ implies $\mathrm{ns}\left({ }^{*} X\right)=\mathrm{st}^{-1}(X)$ $\in L(* \mu, * \mathscr{B}))$.

Corollary 11 together with Corollary 3(iii) implies directly that each finite $\tau$-smooth Borel-measure in a complete metric space is Radon and therefore we obtain the well-known result that each finite Borel-measure in a Polish space is Radon.

Our next result deals with Baire- instead of Borel-measures.

12. TheOREM. Let $(X, \mathscr{T})$ be a completely regular Hausdorff-space with Baire-algebra $\mathscr{B}_{0}$. Let $\mu \mid \mathscr{B}_{0}$ be a finite Baire-measure. Then:

(i) ${ }^{*} \bar{\mu} \circ \mathrm{st}^{-1} \mid \mathscr{B}_{0}$ is the greatest $\tau$-smooth minorant of $\mu \mid \mathscr{B}_{0}$;

(ii) ${ }^{*} \mu \circ \mathrm{st}^{-1} \mid \mathscr{B}_{0}$ is the greatest Radon-minorant of $\mu \mid \mathscr{B}_{0}$;

(iii) $\bar{\mu} \mid \mathscr{B}_{0} \tau$-smooth $\Leftrightarrow \mu\left|\mathscr{B}_{0}={ }^{*} \bar{\mu} \circ \mathrm{st}^{-1}\right| \mathscr{B}_{0}$;

(iv) $\mu \mid \mathscr{B}_{0}$ Radon $\Leftrightarrow \mu\left|\mathscr{B}_{0}={ }^{*} \mu \circ \mathrm{st}^{-1}\right| \mathscr{B}_{0}$. 
Proof. We shall apply Theorem 4 and Lemma 6 to the system $\mathscr{T}_{1}$ of exact open sets and $\mathscr{B}_{1}=\mathscr{B}_{0}$.

"(iii) $\Leftarrow$ " and "(iv) $\Leftarrow$ " follow at once from Theorem 4(ii) as the restriction of a $\tau$-smooth [Radon-] measure is $\tau$-smooth [Radon] on $\mathscr{B}_{0}$.

As each Baire-measure is regular, we obtain "(iii) $\Rightarrow$ " by Lemma 6 . Now we prove "(iv) $\Rightarrow$ ":

As a Radon-measure is $\tau$-smooth, we have $\mu\left|\mathscr{B}_{0}={ }^{*} \bar{\mu} \circ \mathrm{st}^{-1}\right| \mathscr{B}_{0}$ by (iii). According to Theorem 5 , it suffices to prove $\mathrm{ns}\left({ }^{*} X\right) \in L\left({ }^{*} \mu,{ }^{*} \mathscr{B}_{0}\right)$. As $\mu(X)={ }^{*} \bar{\mu}\left(\mathrm{ns}\left({ }^{*} X\right)\right)$, we have to prove

$$
(+) \quad \sup \left\{{ }^{*} \mu_{L}(B): B \subset \mathrm{ns}\left({ }^{*} X\right), B \in L\left({ }^{*} \mu,{ }^{*} \mathscr{B}_{0}\right)\right\} \geqslant \mu(X) .
$$

As $\mu$ is Radon, for each $\varepsilon>0$ there exists a compact set $K$ with $\mu\left(B_{0}\right) \geqslant \mu(X)-\varepsilon$ for each $B_{0} \in \mathscr{B}_{0}$ with $B_{0} \supset K$. We have by Lemma 3.4.4 of [1] that $\mathrm{st}^{-1}(K)=$ $\cap\left\{{ }^{*} T: K \subset T, T\right.$ exact open $\}$. Hence $\mathrm{st}^{-1}(K) \in L\left({ }^{*} \mu,{ }^{*} \mathscr{B}_{0}\right)$ (use Theorem 1(i)) and we obtain by Theorem 1(ii) that

$$
{ }^{*} \mu_{L .}\left(\mathrm{st}^{-1}(K)\right)=\inf \left\{{ }^{*} \mu_{L}\left({ }^{*} T\right): K \subset T, T \text { exact open }\right\} \geqslant \mu(X)-\varepsilon .
$$

As $\mathrm{st}^{-1}(K) \subset \mathrm{ns}\left({ }^{*} X\right)$, this implies $(+)$.

Now we prove (i) and (ii). Applying Theorem 4(ii) once more, we obtain that ${ }^{*} \bar{\mu} \circ \mathrm{st}^{-1} \mid \mathscr{B}_{0}\left[{ }^{*} \mu \circ \mathrm{st}^{-1} \mid \mathscr{B}_{0}\right]$ is $\tau$-smooth [Radon] and for each exact open set 0

$$
{ }^{*} \underline{\mu}\left(\mathrm{st}^{-1}(0)\right) \leqslant * \bar{\mu}\left(\mathrm{st}^{-1}(0)\right) \leqslant *^{*}\left({ }^{*} 0\right)=\mu(0),
$$

i.e. ${ }^{*} \bar{\mu} \circ \mathrm{st}^{-1} \mid \mathscr{B}_{0}$ is a $\tau$-smooth minorant $\left[{ }^{*} \mu \circ \mathrm{st}^{-1} \mid \mathscr{B}_{0}\right.$ is a Radon-minorant] of $\mu \mid \mathscr{B}_{0}$.

Let $\nu\left|\mathscr{B}_{0} \leqslant \mu\right| \mathscr{B}_{0}$ be a $\tau$-smooth [Radon-] minorant. Then ${ }^{*} \bar{\nu} \circ \mathrm{st}^{-1} \leqslant{ }^{*} \bar{\mu} \circ \mathrm{st}^{-1}$ $\left[{ }^{*} \underline{\nu} \circ \mathrm{st}^{-1} \leqslant{ }^{*} \underline{\mu} \circ \mathrm{st}^{-1}\right]$ and the assertion follows from (iii) [(iv)].

From Theorem 12 we obtain a decomposition for Baire-measures in the same way as we got the decomposition of regular Borel-measures. The standard proof of this decomposition result for Baire-measures is due to Knowles [6].

Now we give a simple nonstandard proof of a famous extension theorem for $\tau$-smooth Baire-measures.

13. CoROllary. Let $(X, \mathscr{T})$ be a completely regular Hausdorff-space. Let $\mu \mid \mathscr{B}_{0}$ be a finite $\tau$-smooth Baire-measure. Then ${ }^{*} \bar{\mu} \circ \mathrm{st}^{-1} \mid \mathscr{B}$ is the unique $\tau$-smooth extension of $\mu \mid \mathscr{B}_{0}$.

Proof. According to Theorem 4(ii) applied to the system $\mathscr{T}_{1}$ of exact open sets, ${ }^{*} \bar{\mu} \circ \mathrm{st}^{-1} \mid \mathscr{B}$ is a $\tau$-smooth Borel-measure. According to Theorem 12(iii), we have that ${ }^{*} \bar{\mu} \circ \mathrm{st}^{-1} \mid \mathscr{B}$ is an extension of $\mu \mid \mathscr{B}_{0}$. The uniqueness is trivial.

For compact spaces the standard proof of the following corollary is due to Kakutani.

14. Corollary. Let $\left(X_{n}, \mathscr{T}_{n}\right), n \in \mathbf{N}$, be regular Hausdorff-spaces and let $P_{n}$ be Radon probability measures on the Borel-fields $\mathscr{B}_{n}$ of $X_{n}$. Then there exists a unique Radon-measure on the Borel-algebra $\mathscr{B}$ of $X_{n \in \mathrm{N}} X_{n}$ which extends the product measure $\times_{n \in \mathrm{N}} P_{n} \mid \times_{n \in \mathrm{N}} \mathscr{B}_{n}$. 
Proof. Let $\mathscr{S}$ be the system of all finite unions of $T_{1} \times \cdots \times T_{n} \times X_{n+1} \times X_{n+2}$ $\times \cdots$ with $T_{i} \in \mathscr{T}_{i}, i=1, \ldots, n$ and $n \in \mathbf{N}$. Then $\mathscr{S}$ is a base for the product topology with $\mathscr{A}:=\sigma(\mathscr{S})=\times_{n \in \mathrm{N}} \mathscr{B}_{n}$. Put $\mu=\times_{n \in \mathrm{N}} P_{n}$. Applying Theorem 4(ii) to $X=\times_{n \in \mathrm{N}} X_{n}, \mathscr{T}$ the regular product topology, and $\nu={ }^{*} \mu$, we obtain that ${ }^{*} \mu \circ \mathrm{st}^{-1} \mid \mathscr{B}$ is a Radon-measure. As $\mu$ is the countable product measure of the Radon probability measures $P_{n}, n \in \mathbf{N}$, we obtain $1=\mu(X)=\sup \{\mu(K): K \subset X$ compact $\}$. Hence ${ }^{*} \mu\left(\operatorname{cpt}\left({ }^{*} X\right)\right)=1$ whence ${ }^{*} \mu_{L}\left(\mathrm{~ns}\left({ }^{*} X\right)\right)=1$, as $\operatorname{cpt}\left({ }^{*} X\right) \subset \mathrm{ns}\left({ }^{*} X\right)$. Hence we obtain by Theorem 4 that ${ }^{*} \mu \circ \mathrm{st}^{-1}\left|\mathscr{B}={ }^{*} \bar{\mu} \circ \mathrm{st}^{-1}\right| \mathscr{B}$, whence Lemma 6(ii) implies ${ }^{*} \underline{\mu} \circ \mathrm{st}^{-1}|\mathscr{A}=\mu| \mathscr{A}$. Thus ${ }^{*} \underline{\mu} \circ \mathrm{st}^{-1} \mid \mathscr{B}$ is a Radon-measure which extends $\mu \mid \mathscr{A}$. The uniqueness of the Radon-extension is trivial.

The classical result of Kakutani for arbitrarily many compact spaces $X_{i}, i \in I$, follows in the same way.

The following example shows that in most of our results the regularity of the underlying topological space cannot be dispensed with.

15. EXAMPLE. We construct a topological Hausdorff-space $(X, \mathscr{T})$ with countable base and a probability measure $\mu$ on the Borel-algebra $\mathscr{B}$, such that

(i) $\mathrm{st}^{-1}(B) \notin L\left({ }^{*} \mu,{ }^{*} \mathscr{B}\right) \cap \mathrm{ns}\left({ }^{*} X\right)$ for some $B \in \mathscr{B}$;

(ii) ${ }^{*} \bar{\mu} \circ \mathrm{st}^{-1} \mid \mathscr{B}$ is no measure;

(iii) ${ }^{*} \mu \circ \mathrm{st}^{-1} \mid \mathscr{B}$ is not Radon.

Let $D \subset X:=[0,1]$ be a set of inner Lebesgue-measure 0 and outer Lebesgue-measure 1. Let $\hat{\mathscr{T}}$ be the classical topology on $X=[0,1]$ with Borel-algebra $\hat{\mathscr{B}}$. Put

$$
\mathscr{T}=\left\{T_{1} \cup\left(T_{2} \cap D\right): T_{1}, T_{2} \in \hat{\mathscr{T}}\right\} .
$$

Then $(X, \mathscr{T})$ is a topological Hausdorff-space with countable base and Borel-algebra

$$
\mathscr{B}=\sigma(\mathscr{T})=\left\{\left(B_{1} \cap D\right) \cup\left(B_{2} \cap \bar{D}\right): B_{1}, B_{2} \in \hat{\mathscr{B}}\right\} .
$$

Define on $\mathscr{B}$ a probability measure $\mu$ by

$$
\mu\left(\left(B_{1} \cap D\right) \cup\left(B_{2} \cap \bar{D}\right)\right)=\lambda\left(B_{1}\right),
$$

where $\lambda$ is the Lebesgue-measure on $\hat{\mathscr{B}}$.

As $\bar{D}$ has outer Lebesgue-measure 1 , the definitions of $\mathscr{T}$ and $\mu$ imply that

$$
\bar{D} \subset T \in \mathscr{T} \Rightarrow \mu(T)=1 .
$$

We prove that

$$
\begin{gathered}
{ }^{*} \bar{\mu} \circ \mathrm{st}^{-1}(D)=1 ; \\
{ }^{*} \bar{\mu} \circ \mathrm{st}^{-1}(\bar{D})=1 ; \\
{ }^{*} \circ \circ \mathrm{st}^{-1}(K)=0 \quad \text { for each } \mathscr{T} \text {-compact } K ; \\
{ }^{*} \mu \circ \mathrm{st}^{-1}(X)=1 .
\end{gathered}
$$

Obviously (1), (2) imply (ii) and hence (i), and (3), (4) imply (iii).

To (1) and (2). Let $C \subset X$ be given. We prove at first that

$$
\mathrm{st}^{-1}(C) \subset B \in *^{*} \mathscr{B} \Rightarrow{ }^{*} \mu_{L}(B) \geqslant \mu(T) \text { for some } C \subset T \in \mathscr{T} \text {. }
$$




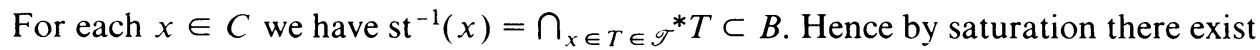
$T_{x} \in \mathscr{T}$ with $x \in T_{x}$ and ${ }^{*} T_{x} \subset B$. Put $T=\bigcup_{x \in C} T_{x}$. Then $C \subset T \in \mathscr{T}$ and we obtain by Theorem 1 that

$$
\begin{aligned}
*^{*} \mu_{L}(B) & \geqslant{ }^{*} \mu_{L}\left(\bigcup_{x \in C} * T_{x}\right)=\sup \left\{{ }^{*} \mu_{L}\left(\bigcup_{x \in E} * T_{x}\right): E \subset C \text { finite }\right\} \\
& =\sup \left\{\mu\left(\bigcup_{x \in E} T_{x}\right): E \subset C \text { finite }\right\}=\mu(T),
\end{aligned}
$$

where the last equality follows, as in a space with countable base each measure is $\tau$-smooth. If $C=D$, then we obtain from (5) that

$$
{ }^{*} \bar{\mu} \circ \mathrm{st}^{-1}(D)=\inf \left\{{ }^{*} \mu_{L}(B): \mathrm{st}^{-1}(D) \subset B \in \mathbb{*}^{*} \mathscr{B}\right\} \underset{(5)}{\geqslant} \mu(D)=1 .
$$

If $C=\bar{D}$, then we obtain from (5) and (0) that

$$
*^{\bar{\mu}} \circ \mathrm{st}^{-1}(\bar{D}) \underset{(5)}{\geqslant} \inf \{\mu(T): \bar{D} \subset T \in \mathscr{T}\} \underset{(0)}{=1} 1 .
$$

This proves (1) and (2).

To (3). Let $K \subset X$ be $\mathscr{T}$-compact. Since $\bar{D}$ is $\mathscr{T}$-closed, $K \cap \bar{D}$ is $\mathscr{T}$-compact, too. Hence $K, K \cap \bar{D}$ are $\hat{\mathscr{T}}$-compact and therefore $\hat{\mathscr{T}}$-closed. Consequently

$$
K \cap D \in \hat{\mathscr{B}} \text {. }
$$

By Theorem 1 we obtain

$$
\begin{aligned}
{ }^{*} \mu \circ \mathrm{st}^{-1}(K) & ={ }^{*} \mu_{L}\left(\bigcap_{K \subset T \in \mathscr{T}} * T\right)=\inf _{K \subset T \in \mathscr{T}} \mu(T) \\
& =\inf _{K \subset T \in \mathscr{T}} \mu(T \cap D)=\mu(K \cap D)=\lambda(K \cap D)=0,
\end{aligned}
$$

since $D$ has inner Lebesgue-measure 0 .

To (4). Let $\widehat{\text { st }}$ be the standard part with respect to the topology $\hat{\mathscr{T}}$. Since $X$ is $\hat{\mathscr{T}}$-compact, we have ${ }^{*} X=\widehat{s t}^{-1}(X)$. As furthermore

$$
\mathrm{st}^{-1}(X)=\left(\widehat{\mathrm{st}}^{-1}(D) \cap * D\right) \cup \widehat{\mathrm{st}}^{-1}(\bar{D})
$$

we obtain $\mathrm{st}^{-1}(X) \supset{ }^{*} D$. As $\mu(D)=1$, this implies (4).

The authors thank the referee for valuable suggestions and for pointing out new literature.

\section{REFERENCES}

1. S. Albeverio, J. E. Fenstad, R. Høegh-Krohn, and T. Lindstrøm, Nonstandard methods in stochastic analysis and mathematical physics, Academic Press, New York, 1986.

2. Robert M. Anderson, Star-finite representations of measure spaces, Trans. Amer. Math. Soc. 271 (1982), 667-687.

3. Robert M. Anderson and Salim Rashid, A nonstandard characterization of weak convergence, Proc. Amer. Math. Soc. 69 (1978), 327-332.

4. Nigel J. Cutland, Nonstandard measure theory and its applications, Bull. London Math. Soc. 15 (1983), 525-589.

5. C. Ward Henson. Analytic sets, Baire sets and the standard part map, Canad. J. Math. 31 (1979), $663-672$ 
6. J. D. Knowles, Measures on topological spaces, Proc. London Math. Soc. (3) 17 (1967), 139-156.

7. Peter A. Loeb, Conversion from nonstandard to standard measure spaces and applications in probability theory, Trans. Amer. Math. Soc. 211 (1975), 113-122.

8. $128-135$

9. . Weak limits of measures and the standard part map, Proc. Amer. Math. Soc. 77 (1979), Analysis and Related Topics, vol. 2 (A. T. Bharucha-Reid, ed.), Academic Press, New York, 1979, pp. $105-142$.

10. Measure spaces in nonstandard models underlying standard stochastic processes, Proc. Internat. Congr. Math., Warzaw, 1983.

11. , A functional approach to nonstandard measure theory, Conference on Modern Analysis and Probability (Beals et al., eds), Amer. Math. Soc., Providence, R.I., 1984.

12. W. A. J. Luxemburg, A general theory of monads, Applications of Model Theory to Algebra, Analysis, and Probability (W. A. J. Luxemburg, ed.), Holt, Rinehart and Winston, New York, 1969, pp. $18-86$.

13. A. Saponakis and M. Sion, On generation of Radon-like measures, Lecture Notes in Math., vol. 1033, Springer-Verlag, Berlin and New York, 1983, pp. 283-294.

14. K. D. Stroyan and W. A. J. Luxemburg, Introduction to the theory of infinitesimals, Academic Press, New York, 1976.

Mathematisches Institut der Universität ZU KOln, Weyertal 86-90, D - 5000 KOln 41, Federal REPUBLIC OF GERMANY

Fachbereich 11, Mathematik, Universität-Gesamthochschule Duisburg, Lotharstr. 65 , D - 4100 Duisburg, Federal Republic of Germany 